Check for updates

Cite this: RSC Adv., 2019, 9, 974

Received 16th September 2018 Accepted 12th December 2018

DOI: $10.1039 / \mathrm{c} 8 \mathrm{ra07696k}$

rsc.li/rsc-advances

\section{Deactivation and regeneration of carbon nanotubes and nitrogen-doped carbon nanotubes in catalytic peroxymonosulfate activation for phenol degradation: variation of surface functionalities $\uparrow$}

\author{
Jifei Hou, Lixia Xu, Yuxiang Han, Yuqiong Tang, Haiqin Wan, Zhaoyi Xu (D) \\ and Shourong Zheng (D) *
}

\begin{abstract}
The reuse, deactivation and regeneration of carbon nanotubes (CNT) and N-doped carbon nanotubes (NCNT) were studied in catalytic peroxymonosulfate (PMS) activation for phenol degradation. The results showed that for catalytic PMS activation, marked deactivation was observed on both CNT and NCNT, resulting in marked variation of the surface functionalities of the catalysts. Catalytic PMS activation led to markedly increased oxygen-containing functionalities and decreased points of zero charge (PZCs) of CNT and NCNT. The catalytic activity of CNT was strongly dependent on the initial PMS concentration but was independent of the initial phenol concentration. Furthermore, the dependency of the CNT activity on the initial PMS concentration closely followed the Langmuir-Hinshelwood model, indicating that the catalytic activation of adsorbed PMS was the rate controlling step. For the used CNT and NCNT, chemical reduction by $\mathrm{NaBH}_{4}$ or thermal treatment regeneration under inert atmosphere could effectively remove surface O-containing functionalities and enhance PZCs, restoring their catalytic activities; meanwhile, the N-containing functionalities of NCNT decreased with regeneration treatment, resulting in a negative impact on catalyst regeneration. The present findings indicate that surface functionalities are closely correlated with catalyst deactivation and regeneration, playing crucial roles in the catalytic activation of PMS.
\end{abstract}

\section{Introduction}

Radical-based advanced oxidation processes (AOPs) are considered to be effective approaches for the abatement of recalcitrant organic pollutants in water. Among AOPs, the Fenton reaction has been widely used in the removal of organic pollutants via an oxidation pathway involving highly reactive hydroxyl radicals $(\cdot \mathrm{OH})$. Notably, hydroxyl radicals are generated only under acidic $\mathrm{pH}$ conditions $(3<\mathrm{pH}<4)$ in the Fenton reaction system, ${ }^{1}$ imposing strong restrictions for its wide application in wastewater treatment. In contrast, sulfate radical $\left(\mathrm{SO}_{4}{ }^{-{ }^{-}}\right.$)-based AOPs (SR-AOPs), which are capable of degrading organic contaminants by highly reactive sulfate radicals in a very wide $\mathrm{pH}$ range of 3 to 10 , are gaining increasing attention. ${ }^{2,3}$

State Key Laboratory of Pollution Control and Resource Reuse, Jiangsu Key Laboratory of Vehicle Emissions Control, School of the Environment, Nanjing University, Nanjing 210093, P. R. China. E-mail: srzheng@nju.edu.cn; Fax:+86-25-89680596; Tel: +86-2589680373

$\dagger$ Electronic supplementary information (ESI) available. See DOI: $10.1039 / \mathrm{c} 8 \mathrm{ra07696k}$
Different methods have been applied to activate persulfates (peroxymonosulfate/PMS or peroxodisulfate/PDS) to produce $\mathrm{SO}_{4}{ }^{--}$, such as heat, ${ }^{4}$ ultraviolet light, ${ }^{5,6}$ ultrasound, ${ }^{7}$ and catalysis. Among these methods, catalysis is extensively used because of its high efficiency and feasibility. In general, metal oxides and carbonaceous materials have been usually used as effective catalysts for persulfate activation. For persulfate activation over metal oxides, $\mathrm{Co}_{3} \mathrm{O}_{4}, \mathrm{Fe}_{2} \mathrm{O}_{3}$ and $\mathrm{MnO}_{2}$ were reported to be active, ${ }^{8-10}$ whereas metal ion leaching concurrently happened during degradation processes, leading to serious secondary pollution. In contrast, carbonaceous materials displayed much stronger advantages over metal oxides due to their high stability toward acid and base in water. Accordingly, different carbonaceous catalysts have been explored for the activation of persulfate. For example, Wang's group systematically explored the catalytic activation on different carbonaceous materials, including carbon nanotubes, hexagonal ordered mesoporous carbon (CMK-3), graphene, nanodiamonds, S- and $\mathrm{N}$-doped graphenes and $\mathrm{N}$-doped nanocarbon materials. ${ }^{11-16} \mathrm{~A}$ variety of active sites of carbon nanomaterials were believed to be capable of activating PMS, such as graphitic carbon structure defect sites, ketonic $(\mathrm{C}=\mathrm{O})$ groups and $\mathrm{N}$-doped functional 
groups. ${ }^{17-22}$ In heterogeneous catalysis, catalyst reuse is essential for practical application. Despite their high catalytic activities, carbonaceous materials usually undergo deactivation during SR-AOPs processes. For example, Duan et al. ${ }^{17}$ observed remarkable deactivation of reduced graphene oxide for phenol degradation; they ascribed the catalyst deactivation to changes in the pore structure and surface chemistry as well as coverage of the catalyst surface by organic intermediates. In parallel, catalyst deactivation was also observed on carbon nanotubes, mesoporous carbon, and N-doped carbon nanotubes. ${ }^{12,16,17}$ However, few studies have systematically discussed the mechanism of catalyst deactivation and regeneration of carbon-based materials in persulfate activation. Accordingly, the variation of the surface functionalities and their roles during catalyst deactivation and regeneration remain unclear.

In the present study, we used carbon nanotubes (CNTs) and $\mathrm{N}$-doped carbon nanotubes (NCNTs) with stable structures as model catalysts to systematically study the roles of surface functionalities in the deactivation and regeneration of carbonbased catalysts during catalytic PMS activation. The fresh, used and regenerated catalysts were characterized using $\mathrm{N}_{2}$ adsorption, Raman spectroscopy, X-ray photoelectron spectroscopy (XPS), Boehm titration and point of zero charge (PZC) measurements. The catalytic performance of the fresh, used and regenerated catalysts was evaluated via activating PMS for phenol degradation.

\section{Experimental}

\subsection{Catalyst preparation}

Preparation of fresh CNT and NCNT. Multiwalled carbon nanotubes (CNT > 97\%) were obtained from Nanotech Port Co. (Shenzhen, Guangdong Province, China). The product information from the manufacturer indicated that the multiwalled CNT had outer diameters in the range of 40 to $60 \mathrm{~nm}$. The sample had a specific surface area of around $100 \mathrm{~m}^{2} \mathrm{~g}^{-1}$ and a pore volume of $0.25 \mathrm{~cm}^{3} \mathrm{~g}^{-1}$ (see Table S1, ESI $\dagger$ ). The sample was pretreated to remove trace metals and amorphous carbon according to a previously reported method. ${ }^{23}$ Briefly, the CNT sample was first calcined at $350{ }^{\circ} \mathrm{C}$ in air for $30 \mathrm{~min}$ with a ramping rate of $5.0{ }^{\circ} \mathrm{C} \mathrm{min}^{-1}$. Then, $1 \mathrm{~g}$ of treated sample was suspended in a mixture of $28 \mathrm{~mL}$ of NaClO solution (chlorine content $>5 \%$ ) and $12 \mathrm{~mL}$ of distilled water; the mixture was heated in a water bath at $85{ }^{\circ} \mathrm{C}$ for $3 \mathrm{~h}$. After cooling to room temperature, the sample was repeatedly washed until neutral and was recovered by filtration using a $0.45 \mu \mathrm{m}$ nylon filter membrane. To remove metal impurities, the sample was further mixed with $2 \% \mathrm{HNO}_{3}$ solution under stirring for $24 \mathrm{~h}$ and was then separated by filtration, followed by washing until neutral and drying at $80{ }^{\circ} \mathrm{C}$ for $12 \mathrm{~h}$ in vacuum. To investigate the influence of calcination, the resulting material was calcined under $\mathrm{N}_{2}$ using a porcelain ark at different calcination temperatures in a horizontal tube furnace. The special carbonization conditions were as follows. CNT samples were

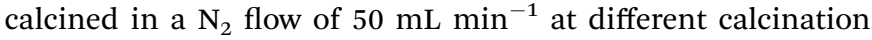
temperatures (i.e., $550{ }^{\circ} \mathrm{C}, 750{ }^{\circ} \mathrm{C}$ and $950{ }^{\circ} \mathrm{C}$ ) for $2 \mathrm{~h}$ with a ramping rate of $5{ }^{\circ} \mathrm{C} \min ^{-1}$ and were labeled as CNT- $X$, where $X$ is the calcination temperature $\left({ }^{\circ} \mathrm{C}\right)$.

To prepare N-doped CNT, $2.0 \mathrm{~g}$ of pretreated CNT was suspended in $300 \mathrm{~mL}$ of mixed acid consisting of concentrated $\mathrm{H}_{2} \mathrm{SO}_{4}$ and $\mathrm{HNO}_{3}(1: 1, \mathrm{v} / \mathrm{v})$; the CNT was refluxed at $80{ }^{\circ} \mathrm{C}$ in a water bath for $5 \mathrm{~h}$. After cooling to room temperature, the sample was collected by filtration, washed with distilled water until neutral, and dried at $80{ }^{\circ} \mathrm{C}$ for $12 \mathrm{~h}$ in vacuum. Then, $2.0 \mathrm{~g}$. of acid-treated sample was suspended in $20 \mathrm{~mL}$ of distilled water containing $20 \mathrm{~g}$ of urea; the suspension was subjected to ultrasonic treatment for $20 \mathrm{~min}$. After water evaporation at $60{ }^{\circ} \mathrm{C}$, the resulting composite material was heat-treated under the same conditions as CNT- $X$. After cooling to room temperature, NCNT- $X$ was washed using distilled water to remove unreacted impurities and dried at $80{ }^{\circ} \mathrm{C}$ for $12 \mathrm{~h}$ in vacuum.

Preparation of used CNT and NCNT. To study the deactivation mechanism, CNT-750 and NCNT-750 were selected and reused 4 times in the presence of phenol. After each reaction, the reused catalysts were collected using a $0.45 \mu \mathrm{m}$ filter, washed with deionized water and ethanol to remove the adsorbed organic intermediates, and then dried at $80{ }^{\circ} \mathrm{C}$ for $12 \mathrm{~h}$ in vacuum. For the reused catalysts obtained in the presence of phenol, the catalysts were labeled as U-CNT- $Y$ and UNCNT- $Y$, respectively, where $Y$ denotes the number of catalyst reuse cycles. To investigate the influence of organic intermediates covering the catalyst surface, CNT-750 and NCNT-750 were reused 2 times in the absence of phenol. After each reaction, the reused catalysts were collected using $0.45 \mu \mathrm{m}$ filter, washed with deionized water, and then dried at $80{ }^{\circ} \mathrm{C}$ for $12 \mathrm{~h}$ in vacuum; the obtained catalysts were denoted as O-CNT- $Y$ and O-NCNT- $Y$.

Preparation of regenerated CNT and NCNT. The used catalysts were regenerated using different approaches, including thermal treatment and chemical reduction with $\mathrm{NaBH}_{4}$ as the reductant. For thermal regeneration, the used catalyst was heated in a $\mathrm{N}_{2}$ flow of $50 \mathrm{~mL} \mathrm{~min}^{-1}$ at a preset temperature (i.e., $350{ }^{\circ} \mathrm{C}, 550{ }^{\circ} \mathrm{C}$ or $750{ }^{\circ} \mathrm{C}$ ) for $2 \mathrm{~h}$ with a ramping rate of $5{ }^{\circ} \mathrm{C} \min ^{-1}$. The regenerated catalyst was denoted as T-CNT- $X$ or T-NCNT- $X$, where $X$ is the thermal regeneration temperature $\left({ }^{\circ} \mathrm{C}\right)$. Chemical reduction was also used to regenerate the catalyst. Briefly, $0.2 \mathrm{~g}$ of used catalyst was suspended in $200 \mathrm{~mL}$ of ethanol under ultrasonic treatment for $20 \mathrm{~min}$. The suspension system was cooled in an ice bath, and $1.0 \mathrm{~g}$ of $\mathrm{NaBH}_{4}$ was added; this mixture was vigorously stirred for $12 \mathrm{~h}$. The regenerated catalyst was recovered by filtration, washed with water and acetone several times, and dried at $80{ }^{\circ} \mathrm{C}$ under vacuum for $12 \mathrm{~h}$. The regenerated U-CNT- 1 and U-NCNT-1 were denoted as RCNT and R-NCNT, respectively.

\subsection{Catalyst characterization}

The specific surface areas of the samples were measured using the $\mathrm{N}_{2}$ adsorption method on a Micromeritics ASAP 2020 instrument (Micromeritics Instrument Co., Norcross, GA), and calculated by the Brunauer-Emmett-Teller (BET) equation in the relative pressure $\left(P / P_{0}\right)$ range of 0.05 to 0.3 . Raman spectra of the CNT and NCNT samples were recorded on a Jobin Yvon LabRam spectrometer (HR-800) with a laser excitation 
wavelength of $514 \mathrm{~nm}$. The compositions and chemical states of the samples were determined by XPS performed on an ESCALAB 250 instrument (Thermo-VG Scientific, USA) equipped with a monochromatized $\mathrm{Al} \mathrm{K} \alpha$ excitation source $(h \nu=1486.6 \mathrm{eV})$. The binding energy values were calibrated using the C1s peak (284.6 eV).

The points of zero charge (PZCs) of the fresh, used and regenerated catalysts were measured using the potentiometric mass titration method. ${ }^{24,25}$ Briefly, $0.1 \mathrm{~g}$ of the sample was mixed with $20 \mathrm{~mL}$ of $0.01 \mathrm{M} \mathrm{NaCl}$ solution at $25{ }^{\circ} \mathrm{C}$, to which $0.2 \mathrm{~mL}$ of 1.0 $\mathrm{M} \mathrm{NaOH}$ was added. The mixture was equilibrated for $24 \mathrm{~h}$ and was then titrated with $0.5 \mathrm{M} \mathrm{HNO}_{3}$ solution under continuous bubbling with a $\mathrm{N}_{2}$ flow $\left(100 \mathrm{~mL} \min ^{-1}\right)$. The $\mathrm{pH}$ was recorded after each addition of the acidic solution. A blank solution was titrated using the above described procedure.

The concentrations of oxygen-containing groups of the samples were quantified using Boehm titration. Briefly, $0.5 \mathrm{~g}$ of sample was added to $25 \mathrm{~mL}$ of $0.025 \mathrm{M}$ base or acid solution (e.g., $\mathrm{NaHCO}_{3}, \mathrm{Na}_{2} \mathrm{CO}_{3}, \mathrm{NaOH}$, and $\mathrm{HCl}$ ), and the mixture was stirred for $48 \mathrm{~h}$. After filtration using a $0.45 \mu \mathrm{m}$ filter, $10 \mathrm{~mL}$ of filtrate was placed in a beaker. The base solutions of $\mathrm{NaHCO}_{3}$ and $\mathrm{NaOH}$ were acidified with $20 \mathrm{~mL}$ of $0.025 \mathrm{M} \mathrm{HCl}$ solution, and $\mathrm{Na}_{2} \mathrm{CO}_{3}$ solution was acidified with $30 \mathrm{~mL}$ of $0.025 \mathrm{M} \mathrm{HCl}$. The acidified solutions were back-titrated with $0.025 \mathrm{M} \mathrm{NaOH}$ solution using a Metrohm 877 Titrino Plus automated titrator (Metrohm Ltd., Switzerland). All solutions were titrated in duplicate and the averages were used. The standardization of the $\mathrm{NaOH}$ solution was conducted with potassium hydrogen phthalate as the primary standard and phenolphthalein as the indicator. The concentration of the $\mathrm{HCl}$ solution was determined using standardized $\mathrm{NaOH}$ solution. All titration processes were carried out under $\mathrm{N}_{2}$ gas protection to exclude the effects of $\mathrm{CO}_{2}$ from the air.

\subsection{PMS activation for phenol degradation}

Phenol oxidation by PMS activation on the catalysts was conducted in a $250 \mathrm{~mL}$ three-necked flask reactor under atmospheric pressure at $25 \pm 0.5{ }^{\circ} \mathrm{C}$ with the reaction temperature stabilized by a water-bath (SDC-6, Scientz Co., China). Briefly, $20 \mathrm{mg}$ of catalyst was suspended in $198 \mathrm{~mL}$ of $10 \mathrm{mM}$ phosphate buffer solution ( $\mathrm{pH}$ 7.0). Then, $2 \mathrm{~mL}$ of $10.6 \mathrm{mM}$ phenol stock solution was added, and the mixture was vigorously stirred for $40 \mathrm{~min}$ in order to reach adsorption equilibrium. Preliminary experiments showed that phenol adsorption on the catalysts varied by $1 \%$ to $2 \%$ (data not presented). The catalytic reaction was started by adding $8 \mathrm{mM}$ of PMS. Samples were taken at preset intervals, and the catalyst particles were rapidly separated by filtration using a $0.45 \mu \mathrm{m}$ filter. The filtrates were added to vials containing $0.5 \mathrm{~mL}$ of methanol. The residual concentration of phenol in the filtrate was analyzed by high performance liquid chromatography (Agilent 1200 Series HPLC system). Phenol was separated on an SB-C18 column $(150 \times 4.6$ $\mathrm{mm}, 5 \mu \mathrm{m})$ with $30 \%$ acetonitrile and $70 \%$ water $(\mathrm{v} / \mathrm{v})$ as the mobile phase at a flow rate of $0.8 \mathrm{~mL} \min ^{-1}$ and detected by a UV detector with a wavelength of $270 \mathrm{~nm}$. Standard curves with six concentrations were used to determine the phenol concentrations. The catalyst activity was evaluated in terms of the initial activity, which was defined as the specific removal rate of phenol within the initial $5 \mathrm{~min}$. Preliminary experiments indicated that mass transfer limitations were not observed under our experimental conditions, and the experiments had very high repeatability with experimental errors below $5 \%$, as confirmed by two separate runs of phenol degradation on CNT750 (the results are presented in Fig. S1 and S2, ESI $\dagger$ ).

\section{Results and discussion}

\subsection{Catalyst characterization}

The Raman spectra of the CNT and NCNT samples are compiled in Fig. 1. The samples exhibited very strong $G$ and $D$ bands at 1570 and $1331 \mathrm{~cm}^{-1}$, respectively. The $\mathrm{G}$ band is indicative of the stretching vibration from carbon atoms with $\mathrm{sp}^{2}$ bonds in a two-dimensional hexagonal lattice, whereas the $\mathrm{D}$ band is assigned to the vibration of carbon atoms with dangling bonds in plane terminations. The ratios of $\mathrm{D}$ band strength to $\mathrm{G}$ band strength $\left(I_{\mathrm{D}} / I_{\mathrm{G}}\right)$ were calculated to be $1.06,1.17$ and 1.29 for CNT-750, U-CNT-1 and T-CNT-750, respectively, reflecting that the surface defect content of CNT increased after the PMS activation reaction and thermal regeneration. Similar trends were presented by the NCNT samples, with $I_{\mathrm{D}} / I_{\mathrm{G}}$ ratios of 1.15 , 1.21 and 1.23 for NCNT-750, U-NCNT-1 and T-NCNT-750, respectively.

The PZCs of the fresh, used and regenerated catalysts were determined using the potentiometric mass titration method, and the results are presented in Table 1 . For the CNT series, the PZC values of CNT-750 and U-CNT-1 were determined to be 7.3 and 3.7, respectively. The markedly lower PZC of U-CNT-1 than of CNT-750 is likely due to the surface oxidation of CNT during the phenol degradation process. The PZCs of T-CNT-350, TCNT-550 and T-CNT-750 were 5.9, 7.2 and 7.6, respectively, suggesting that thermal treatment at higher temperatures resulted in more markedly increased PZCs of the regenerated catalyst. It was previously reported that the O-containing groups of carbonaceous materials were susceptible to decomposition upon thermal treatment. ${ }^{26}$ Furthermore, Menendez et $a .^{27}$ studied the influence of thermal treatment on the surface groups of activated carbon and concluded that base sites could be formed at the expense of acidic O-containing groups upon thermal treatment; increasing the thermal treatment temperature resulted in a high concentration of base sites. Hence, the
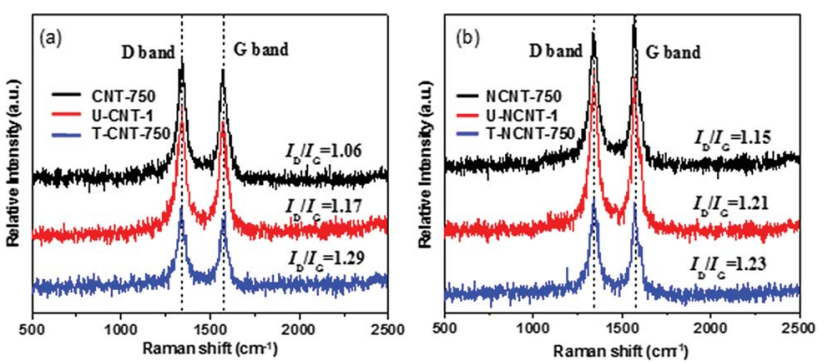

Fig. 1 Raman spectra of fresh, used and regenerated samples: (a) CNT and (b) NCNT. 
Table 1 C, O, and N contents of CNT and NCNT samples determined by XPS

\begin{tabular}{|c|c|c|c|c|c|c|c|c|}
\hline Sample & \multicolumn{2}{|l|}{ XPS } & PZC & Sample & \multicolumn{3}{|l|}{ XPS } & PZC \\
\hline U-CNT-1 & 96.50 & 3.50 & 3.7 & U-NCNT-1 & 93.08 & 4.54 & 2.38 & 5.4 \\
\hline T-CNT-350 & 97.00 & 2.99 & 5.9 & T-NCNT-350 & 93.65 & 4.05 & 2.30 & 6.6 \\
\hline T-CNT-550 & 97.26 & 2.73 & 7.2 & T-NCNT-550 & 94.80 & 3.30 & 1.90 & 7.4 \\
\hline & \multirow{2}{*}{97.09} & \multirow{2}{*}{2.90} & \multirow{2}{*}{6.2} & NCNT-550 & 88.76 & 3.24 & 7.98 & $\mathrm{ND}^{b}$ \\
\hline & & & & NCNT-950 & 95.86 & 2.08 & 2.05 & $\mathrm{ND}^{b}$ \\
\hline
\end{tabular}

${ }^{a}$ Below detection level. ${ }^{b}$ Not determined.

gradually increased PZCs of the catalysts with increasing thermal treatment temperature can be ascribed to the removal of O-containing functionalities and the formation of surface base sites (see more results and discussion below). Notably, the catalyst regenerated at $750{ }^{\circ} \mathrm{C}$ had a slightly higher PZC than the fresh catalyst, despite their thermal treatment at identical temperatures. This can be attributed to the formation of new $\mathrm{O}$ containing groups on the surface of the CNTs during PMS activation (see the XPS and Boehm titration results below), and these groups decomposed upon thermal regeneration, resulting in new Lewis base sites. In comparison with thermal treatment regeneration, reduction regeneration using $\mathrm{NaBH}_{4}$ led to a moderate increase of the catalyst PZC to 6.2.

Similar results were observed on the NCNT samples. The PZC of fresh NCNT-750 was 7.9, higher than that of fresh CNT750, likely due to the presence of N-containing base groups (e.g., pyridinic nitrogen). Application of NCNT-750 in PMS activation also led to a marked decrease of PZC from 7.9 to 5.4 after 1 catalyst reuse cycle. Increasing the thermal regeneration temperature from $350{ }^{\circ} \mathrm{C}$ to $750{ }^{\circ} \mathrm{C}$ led to an increase of the PZC of NCNT-750 from 6.6 to 8.4. In parallel, the PZC of the catalyst increased to 6.6 upon chemical reduction by $\mathrm{NaBH}_{4}$.

XPS analysis was conducted to elucidate the surface properties of the catalysts, and the contents of $\mathrm{C}, \mathrm{O}$ and $\mathrm{N}$ are listed in Table 1. For the CNT samples, the surface O content in UCNT-1 was determined to be 3.50 at\%, higher than that of CNT-750 (2.44 at\%); this is indicative of increased surface Ocontaining groups due to surface oxidation after the reaction. For the regenerated CNTs, the O contents of T-CNT-350, T-CNT550 and T-CNT-750 were 2.99 at\%, 2.73 at\%, and 2.39 at\%, respectively, indicating that thermal regeneration of the used catalysts led to marked decreases in their surface $\mathrm{O}$ contents. In comparison with $\mathrm{NaBH}_{4}$ reduction, a more marked decrease of O-content could be achieved upon thermal regeneration. Additionally, thermal regeneration at a higher temperature resulted in a lower O content. Notably, the C1s spectra of all the samples displayed very strong non-symmetry, implying that their carbon surfaces are composed of multiple species. Hence, the C1s spectra of the fresh, used, and regenerated CNTs were deconvoluted, and the results are compared in Fig. 2a and Table 2. For CNT-750, the C1s spectrum consisted of four peaks with binding energies of 284.3, 285.5, 287.3 and $288.7 \mathrm{eV}$, assigned to carbon atoms from aromatic rings and hydroxyl/ether, carbonyl and carboxyl groups, respectively. In comparison with CNT-750, increased contents of $\mathrm{C}$ atoms with $\mathrm{O}$-functionalities were observed on U-CNT-1 except for the carbon atoms with carbonyl groups, which can be attributed to the conversion of carbonyl carbons to carboxyl carbons via surface oxidation during PMS activation. ${ }^{28}$ Thermal regeneration of U-CNT-1 led to a marked increase of the carbon atom content from aromatic rings and decreases of the carbon atom contents from hydroxyl/ether and carboxyl groups. Furthermore, the content of carbonyl carbon atoms in T-CNT-750 was slightly higher than that of CNT-750, which is indicative of the formation of additional carbonyl groups after the oxidation and regeneration cycle.

For NCNT-750, the surface N content was 2.56 at\% (see Table 1 ), which is indicative of successful surface N-doping of CNT. Similar to CNT-750, catalytic PMS activation led to an increase of the surface O-content from 2.66 at\% to 4.54 at\% due to surface oxidation, whereas the surface $\mathrm{N}$ contents remained nearly constant, reflecting the relatively high stability of $\mathrm{N}$ -
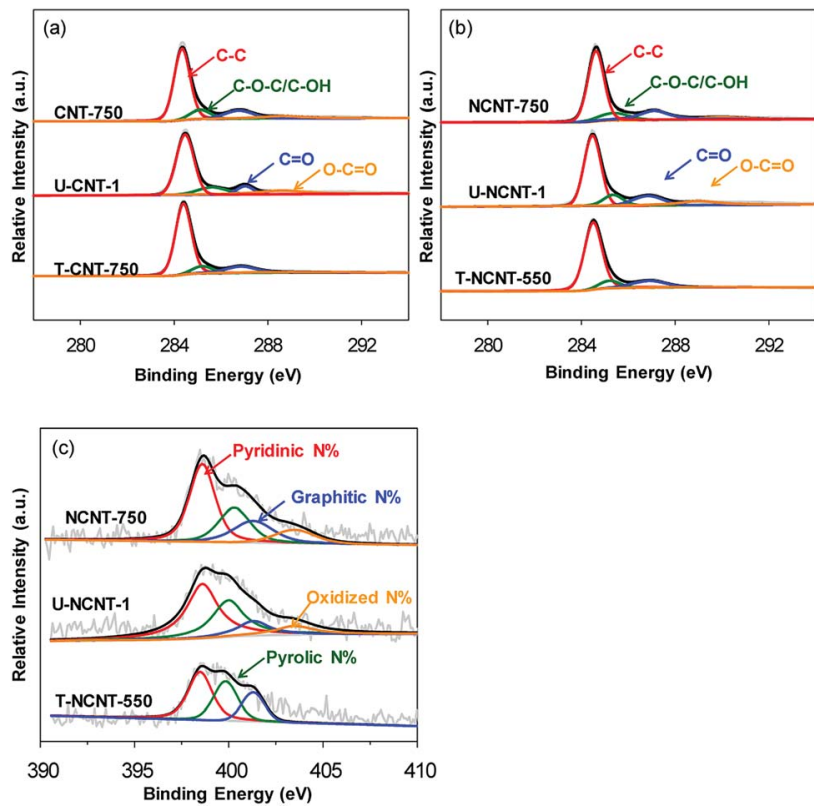

Fig. 2 C1s spectra of (a) the CNT samples and (b) the NCNT samples; (c) N1s spectra of the NCNT samples. 
Table 2 Relative integrated intensities of the deconvoluted C1s and N1s XPS spectra of the catalysts

\begin{tabular}{|c|c|c|c|c|c|c|c|c|}
\hline Sample & \multicolumn{4}{|l|}{ C1s } & \multicolumn{4}{|l|}{ N1s } \\
\hline CNT-750 & 69.3 & 10.4 & 12.7 & 7.6 & - & - & - & - \\
\hline U-CNT-1 & 63.2 & 12.7 & 7.9 & 16.2 & - & - & - & - \\
\hline T-CNT-750 & 73.9 & 8.7 & 14.0 & 3.4 & - & - & - & - \\
\hline T-NCNT-550 & 76.0 & 9.2 & 13.7 & 1.1 & 45.7 & 33.4 & 20.9 & - \\
\hline
\end{tabular}

containing functionalities towards surface oxidation. Again, thermal regeneration resulted in decreased O-content, and the O-content decreased with increasing thermal treatment temperature. Notably, the N contents of T-NCNT-350 and TNCNT-550 were 2.30 and 1.90 at\%, respectively, much lower than those of NCNT-750. Additionally, the N content of T-NCNT750 was even below the detection level, reflecting that surface Ncontaining functionalities were vulnerable to thermal treatment and that thermal treatment at $750{ }^{\circ} \mathrm{C}$ led to nearly complete loss of surface N. The C1s and N1s spectra of the NCNT samples were also deconvoluted, and the results are presented in Fig. $2 \mathrm{~b}$ and c, and Table 2. Again, catalytic PMS activation led to increased contents of hydroxyl and carboxyl carbon atoms but also led to decreased carbonyl and aromatic carbon atoms. Thermal treatment regeneration at $750{ }^{\circ} \mathrm{C}$ resulted in markedly decreased surface functionalities. For fresh NCNT-750, pyridinic, pyrrolic, graphitic and oxidized $\mathrm{N}$ species were identified, and their contents were calculated to be $45.8 \%, 24.0 \%$, $21.5 \%$ and $8.7 \%$ (see Table 2), respectively. Upon catalytic PMS activation, the contents of pyridinic and graphitic $\mathrm{N}$ decreased while the contents of pyrrolic and oxidized $\mathrm{N}$ increased. Thermal regeneration at $550{ }^{\circ} \mathrm{C}$ led to a marked decrease of oxidized $\mathrm{N}$, and thermal treatment at $750{ }^{\circ} \mathrm{C}$ removed all $\mathrm{N}$ containing functionalities.

These results were confirmed by Boehm titration (as listed in Table 3). For the CNT samples, CNT-750 had a very low concentration of O-containing groups $\left(0.033 \mathrm{mmol} \mathrm{g}^{-1}\right)$, while the concentration of O-containing groups of U-CNT-1 was $0.12 \mathrm{mmol} \mathrm{g}^{-1}$, much higher than that of CNT-750. Particularly, the concentration of carboxylic groups increased from $0.018 \mathrm{mmol} \mathrm{g}^{-1}$ to $0.051 \mathrm{mmol} \mathrm{g}^{-1}$ after one cycle of catalyst reuse, which is indicative of apparent surface oxidation of CNT. Concurrently, the content of base sites markedly decreased from $0.12 \mathrm{mmol} \mathrm{g}^{-1}$ to $0.054 \mathrm{mmol} \mathrm{g}^{-1}$. Upon thermal regeneration, substantial changes in the concentrations of $\mathrm{O}$ containing groups were observed on used CNT-750. For UCNT-1, the concentration of O-containing groups decreased from $0.12 \mathrm{mmol} \mathrm{g}^{-1}$ to $0.032 \mathrm{mmol} \mathrm{g}^{-1}$ and the concentration of base groups increased from $0.054 \mathrm{mmol} \mathrm{g}^{-1}$ to $0.078 \mathrm{mmol} \mathrm{g}^{-1}$ after thermal regeneration at $750^{\circ} \mathrm{C}$ for $2 \mathrm{~h}$. In comparison with U-CNT-1, markedly decreased carboxylic groups were observed on T-CNT-550 and T-CNT-750, whereas marked removal of lactone groups was only observed on T-CNT-750; this is indicative of the varied thermal stability of O-containing functionalities. Previous studies showed that after thermal treatment, carboxylic groups were vulnerable and decomposed below $250{ }^{\circ} \mathrm{C}$, while hydroxyl and lactone groups were very stable and only decomposed above $600{ }^{\circ} \mathrm{C} .{ }^{29-31}$ Additionally, thermal treatment led to the decomposition of O-containing functionalities by releasing $\mathrm{CO}$ or $\mathrm{CO}_{2}$, giving rise to surface defect sites and oxygen-free Lewis base sites. ${ }^{27}$ Compared with CNT-750, NCNT-750 had a much lower concentration of carboxylic groups but a much higher concentration of basic groups; this is likely due to the formation of pyridinic and pyrrolic $\mathrm{N}$ species at the expense of carboxylic groups during the N-doping process. Accordingly, Arrigo et al..$^{32}$ studied the surface structure of $\mathrm{N}$ doped CNT and concluded that N-doping occurred preferentially on carboxylic sites. Hence, the markedly higher PZC of

Table 3 Surface group contents determined by Boehm titration

\begin{tabular}{llllll}
\hline Sample & $\begin{array}{l}\text { Carboxylic groups } \\
\left(\mathrm{mmol} \mathrm{g}^{-1}\right)\end{array}$ & $\begin{array}{l}\text { Lactone groups } \\
\left(\mathrm{mmol} \mathrm{g}^{-1}\right)\end{array}$ & $\begin{array}{l}\text { Hydroxy groups } \\
\left(\mathrm{mmol} \mathrm{g}^{-1}\right)\end{array}$ & $\begin{array}{l}\text { Total acidic groups } \\
\left(\mathrm{mmol} \mathrm{g}^{-1}\right)\end{array}$ & $\begin{array}{l}\text { Total basic groups/sites } \\
\left(\mathrm{mmol} \mathrm{g}^{-1}\right)\end{array}$ \\
\hline CNT-750 & 0.018 & 0.013 & 0.002 & 0.033 & 0.120 \\
U-CNT-1 & 0.051 & 0.061 & 0.004 & 0.120 & 0.054 \\
T-CNT-350 & 0.020 & 0.053 & 0.005 & 0.078 & 0.059 \\
T-CNT-550 & 0.005 & 0.057 & 0.004 & 0.066 & 0.063 \\
T-CNT-750 & 0.006 & 0.026 & - & 0.032 & 0.078 \\
NCNT-750 & - & 0.025 & 0.031 & 0.056 & 0.093 \\
U-NCNT-1 & 0.073 & 0.150 & 0.009 & 0.230 & 0.100 \\
T-NCNT-350 & 0.028 & 0.130 & 0.003 & 0.130 & 0.120 \\
T-NCNT-550 & 0.013 & 0.120 & - & 0.063 & 0.130 \\
T-NCNT-750 & 0.009 & 0.054 & - & &
\end{tabular}


NCNT-750 can be ascribed to its much lower content of carboxylic groups and higher content of basic groups compared to CNT-750. In parallel, regeneration of U-NCNT-1 led to markedly decreased acidic group content and to increased content of base groups.

Moreover, the specific surface areas $\left(S_{\mathrm{BET}}\right)$ and pore volumes $\left(V_{\mathrm{t}}\right)$ of fresh, used and regenerated CNT and NCNT catalysts were detected by $\mathrm{N}_{2}$ adsorption curves and are shown in Table $\mathrm{S} 1 . \dagger$ The $S_{\mathrm{BET}}$ of the CNT series catalysts was $98 \pm 4 \mathrm{~m}^{2} \mathrm{~g}^{-1}$, with a $V_{\mathrm{t}}$ of around $0.28 \pm 0.05 \mathrm{~cm}^{3} \mathrm{~g}^{-1}$. The $S_{\mathrm{BET}}$ of the NCNT series catalysts was $110 \pm 5 \mathrm{~m}^{2} \mathrm{~g}^{-1}$ with $V_{\mathrm{t}}$ of around $0.28 \pm 0.02 \mathrm{~cm}^{3}$ $\mathrm{g}^{-1}$. These results indicate that the reuse and regeneration processes play minor roles in the structures of CNT and NCNT.

\subsection{Catalytic PMS activation for phenol degradation}

The catalytic PMS activation for phenol degradation on CNT and NCNT catalysts is shown in Fig. 3a. The catalytic activities of the fresh catalysts were found to be dependent on the activation temperature. For example, increasing the calcination temperature from $550{ }^{\circ} \mathrm{C}$ to $950{ }^{\circ} \mathrm{C}$ led to gradually increased initial activities from 0.028 to $0.041 \mathrm{mmol} \mathrm{g}_{\text {cat }}{ }^{-1} \mathrm{~min}^{-1}$ for the CNT catalysts. Previous studies showed that surface $\mathrm{C}=\mathrm{O}$ groups and $\mathrm{sp}^{2}$ hybrid and Lewis basic sites (defect sites) of carbonaceous catalysts are capable of acting as active sites for catalytic PMS activation. ${ }^{\mathbf{1 2 , 1 6}}$ Increasing the thermal treatment temperature resulted in increased surface basicity and graphite content, which likely accounts for the enhanced catalytic activity of CNT for phenol degradation. For NCNT, increasing the calcination temperature to $950{ }^{\circ} \mathrm{C}$ resulted in sharply decreased catalytic activity due to the loss of nitrogen at this high temperature (see Table 1). Considering the balance between CNT and NCNT, all fresh catalysts were activated at $750{ }^{\circ} \mathrm{C}$ in our study.
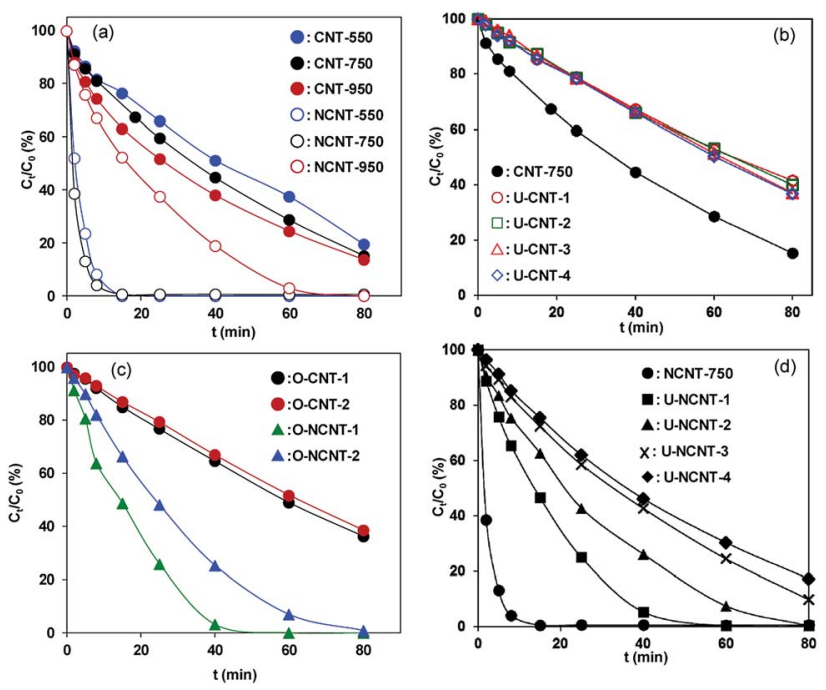

Fig. 3 Phenol degradation on (a) CNT and NCNT catalysts with different calcination temperatures, (b) fresh and used CNT-750, (c) pre-oxidation CNT-750 and NCNT-750 catalysts prepared in the absence of phenol, and (d) fresh and reused NCNT-750. Reaction conditions: $[\mathrm{PMS}]_{0}=8 \mathrm{mM}$; $[\text { phenol }]_{0}=0.106 \mathrm{mM}$; catalyst dosage $=$ $0.1 \mathrm{~g} \mathrm{~L}^{-1}$.
From the viewpoint of practical application, catalyst reuse is essential. Hence, the reuse of CNT-750 in catalytic PMS activation for phenol degradation was studied, and the results are presented in Fig. 3b. After reaction for $40 \mathrm{~min}, 55.3 \%$ phenol was removed on CNT-750 and $32.8 \%$ on U-CNT-1, which is indicative of higher catalytic activity of the fresh catalyst than of the reused catalyst. Accordingly, the initial activities were $0.031 \mathrm{mmol} \mathrm{g}_{\text {Cat }}{ }^{-1} \mathrm{~min}^{-1}$ and $0.01 \mathrm{mmol} \mathrm{g}_{\text {Cat }}{ }^{-1} \mathrm{~min}^{-1}$ for CNT750 and U-CNT-1, respectively, reflecting marked catalyst deactivation after one cycle of catalyst reuse. For multiple-cycle reuses, however, the activities of the used catalysts remained almost constant and further deactivation was not observed. In parallel, catalyst deactivation was previously observed on other carbonaceous catalysts (e.g., activated carbon, mesoporous carbon and CNTs). ${ }^{\mathbf{1 6 , 1 7 , 1 9 , 3 3 , 3 4}}$ The characterization results of XPS and Boehm titration revealed that the contents of O-containing groups substantially increased upon catalyst reuse. Accordingly, much lower PZCs were observed on the used catalysts than on CNT-750, confirming the surface oxidation of CNT-750 after phenol degradation. After one reuse cycle, the activity of U-CNT1 was identical to those of U-CNT-2, U-CNT-3 and U-CNT-4. To exclude the role of residual organic species (i.e., intermediates of phenol degradation) on catalyst deactivation under our conditions, catalytic PMS activation on CNT-750 and NCNT-750 was conducted in the absence of phenol, and the results are presented in Fig. 3c. After PMS activation in the absence of phenol, the initial activity of O-CNT-1 was $0.009 \mathrm{mmol} \mathrm{g}_{\mathrm{Cat}^{-}}$ ${ }^{-1} \min ^{-1}$, almost identical to that of U-CNT-1. Additionally, further deactivation of the catalyst within the consecutive catalytic cycles was not observed. This clearly suggests that the organic reactants and potential intermediate products had no influence on the used catalysts. Thus, excluding the influence of structure and residual organic species on catalyst deactivation, we studied the mechanism of surface functional groups in the process of deactivation and regeneration. Significant catalyst deactivation was also observed on NCNT-750, and the results are shown in Fig. 3d. NCNT-750 exhibited a much higher initial activity of $0.18 \mathrm{mmol} \mathrm{g}_{\mathrm{Cat}}{ }^{-1} \mathrm{~min}^{-1}$ than CNT-750, which can be attributed to the presence of additional N-containing active sites in NCNT. ${ }^{\mathbf{2 0 , 2 1 , 3 5}}$ For catalyst reuse, the initial activity decreased from $0.18 \mathrm{mmol} \mathrm{g}_{\text {Cat }}^{-1} \mathrm{~min}^{-1}$ on NCNT-750 to $0.018 \mathrm{mmol} \mathrm{g}_{\mathrm{Cat}}^{-1} \mathrm{~min}^{-1}$ on U-NCNT-4, which also displayed marked catalyst deactivation. Similarly, the initial activities of U-NCNT-1 and U-NCNT-2 were found to be nearly equal to those of O-NCNT-1 and O-NCNT-2 (results presented in Fig. 3c), confirming that the deactivation was predominantly due to surface oxidation of the catalyst by catalytic PMS activation. Characterization results revealed that catalytic PMS activation led to increased content of oxidized $\mathrm{N}$ species at the expense of pyridinic, graphitic and pyrrolic $\mathrm{N}$ species, reflecting decreased content of catalytic active sites. In contrast to CNT-750, however, NCNT-750 exhibited continuous deactivation within 4 test reuse cycles.

For catalytic PMS activation on carbonaceous catalysts, radical and non-radical mechanisms were previously reported. For example, Lee et al. ${ }^{2,36}$ observed a non-radical mechanism for the degradation of phenolic and pharmaceutical compounds on 
CNTs. In contrast, Duan et al. ${ }^{\mathbf{1 6}}$ studied phenol degradation on $\mathrm{N}$-doped single walled nanotubes and identified a combined radical and non-radical reaction mechanism. To gain more insight into the mechanisms of PMS activation and catalyst deactivation, 5,5-dimethyl-1-pyrroline-N-oxide (DMPO) was used as a probe molecule, and the reaction was monitored using electron paramagnetic resonance (EPR). The EPR spectra under varied reaction conditions are compiled in Fig. 4a. In the absence of catalyst, characteristic peaks from active radicals (e.g., DMPO-OH adducts and $\mathrm{DMPO}-\mathrm{SO}_{4}$ adducts) were not observed. In the presence of catalysts, characteristic peaks with an intensity ratio of $1: 2: 1: 2: 1: 2: 1$ were clearly observed, assigned to the DMPO oxidation product, DMPO- $X .^{2,37,38}$ In parallel, the signal of DMPO- $X$ was also identified in PMS
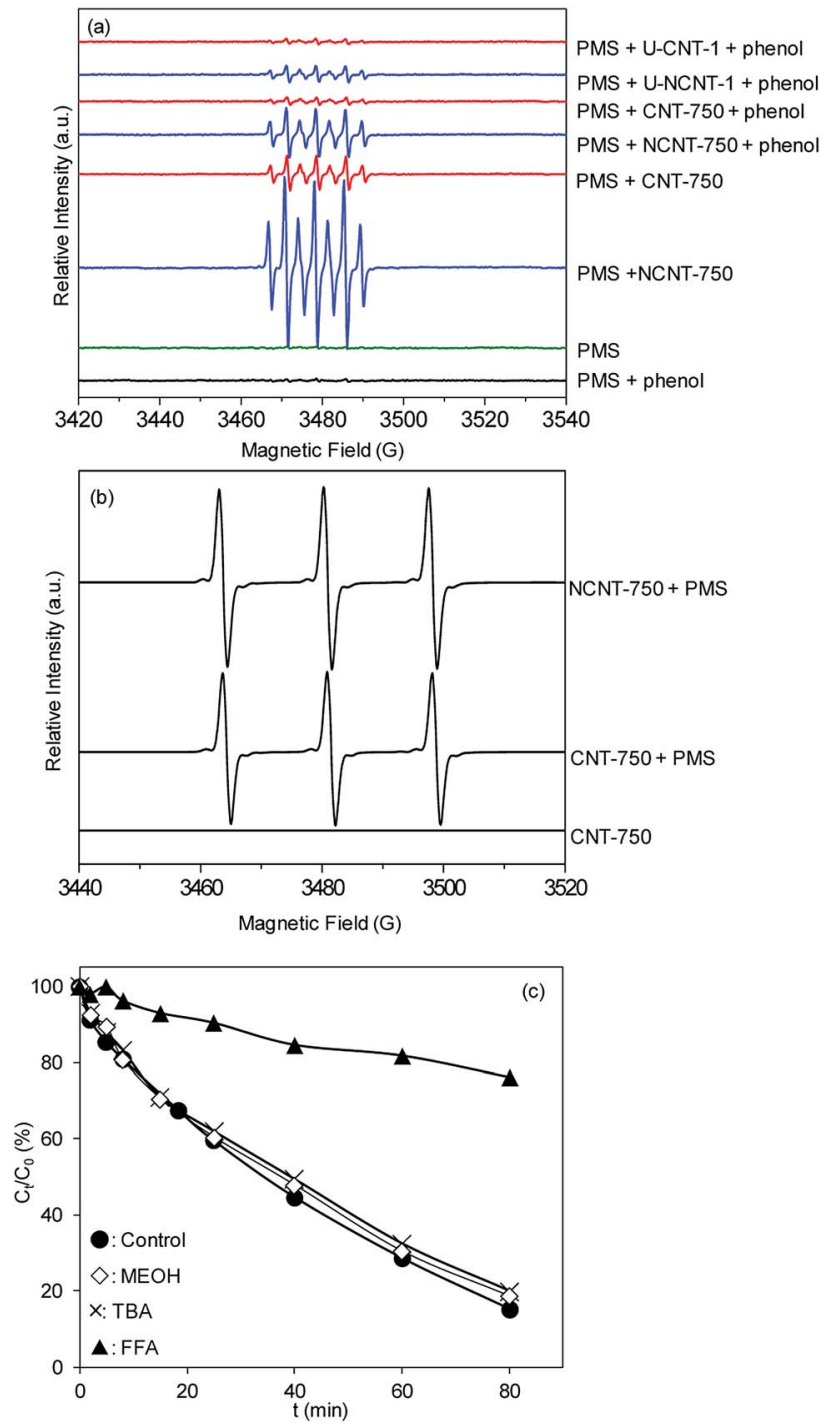

Fig. 4 EPR spectra obtained by trapping with (a) DMPO and (b) TEMP under different conditions and (c) catalytic PMS activation for phenol degradation on CNT-750 in the presence of radical scavengers. EPR conditions: $[\mathrm{DMPO}]_{0}=[\mathrm{TEMP}]_{0}=100 \mathrm{mM}$; $[\text { catalyst }]_{0}=0.1 \mathrm{mg} \mathrm{L}^{-1}$; $[\mathrm{PMS}]_{0}=8 \mathrm{mM} ; \mathrm{pH}=7.4 \pm 0.2(100 \mathrm{mM}$ phosphate buffer); reaction time $=10 \mathrm{~min} ;[\text { phenol }]_{0}=0.106 \mathrm{mM} ;[\mathrm{MEOH}]_{0}=[\mathrm{TBA}]_{0}=[\mathrm{FFA}]_{0}=$ $1.6 \mathrm{M}$. activation on the different catalytic reaction systems. ${ }^{2,38}$ Floyd et $a l .{ }^{39}$ attributed the formation of DMPO- $X$ to direct oxidation by single electron sources. Very recently, many studies have indicated that the appearance of DMPO- $X$ can be ascribed to the proliferation of free radicals. ${ }^{\mathbf{4 0 , 4 1}}$ For PMS activation on NCNTs, similar EPR signals were also observed, while the intensities of the EPR peaks were much stronger; this confirms that PMS activation was more effective on NCNT-750 than on CNT-750. To clarify the impact of catalyst surface oxidation on the activation efficiency, the EPR spectra of PMS activation on the reused catalysts (U-CNT-1 and U-NCNT-1) were also determined. In comparison with fresh catalysts, the intensities of DMPO- $X$ were much weaker, clearly confirming the higher catalytic activities of the fresh catalysts compared to the used catalysts. Additionally, the absence of phenol led to the formation of similar but much stronger EPR peaks than those in the presence of phenol, which can be ascribed to the competitive reaction of phenol with the active species, consequently suppressing the formation of DMPO- $X$. The results were in good agreement with the data of catalytic PMS activation for phenol degradation. TEMP (2,2,6,6-tetramethyl-4-piperidinol) was used as a spintrapping agent to identify other potential active radicals. As shown in Fig. 4b, a three-line spectrum with equal intensities was obtained, which is characteristic of TEMP- ${ }^{1} \mathrm{O}_{2}$ adducts (TEMPO); ${ }^{42,43}$ this is indicative of the presence of ${ }^{1} \mathrm{O}_{2}$ in the PMS/CNT-750 system.

To further verify the activation mechanisms, methanol, $t$ butanol (TBA) and furfuralcohol (FFA) were used as radical scavengers to detect the effects on the degradation of phenol, and the results are presented in Fig. 4c. In parallel, the presence of neither $\mathrm{MEOH}$ nor TBA affected the phenol degradation. Furthermore, the addition of FFA to the PMS/CNT-750 reaction system resulted in decreased phenol removal from $84.7 \%$ to $23.8 \%$ within $80 \mathrm{~min}$, confirming the importance of ${ }^{1} \mathrm{O}_{2}$ in the reaction.

It should be emphasized that in liquid phase catalytic reactions, the adsorption of reactants on the catalyst surface is a prerequisite step and thus plays a crucial role in the catalytic activity of the catalyst. For example, Chen et al. ${ }^{\mathbf{4 4}}$ compared liquid phase catalytic hydrogenation of bromate on Pd catalysts supported on different supports (e.g., $\mathrm{Al}_{2} \mathrm{O}_{3}$, activated carbon (AC) and $\mathrm{SiO}_{2}$ ) and observed a much higher catalytic activity for $\mathrm{Pd} / \mathrm{Al}_{2} \mathrm{O}_{3}$ than for the other catalysts due to strong electrostatic attractive interactions. Inspired by previous results, we assumed that the adsorption of PMS or/and phenol played important roles in phenol degradation. Hence, the influences of the initial concentrations of PMS and phenol on the initial reaction rate of phenol degradation were investigated. For the influence of PMS adsorption, phenol degradation was conducted by varying the initial PMS concentration from 2.5 to $12.0 \mathrm{mM}$ while fixing the phenol concentration at $0.106 \mathrm{mM}$, and the results are compared in Fig. 5a. Increasing the PMS concentration led to an increased initial rate from 0.019 to $0.031 \mathrm{mmol} \mathrm{g}_{\text {Cat }}{ }^{-1} \mathrm{~min}^{-1}$; meanwhile, at PMS concentrations above $8.0 \mathrm{mM}$, the phenol degradation rate remained nearly constant (Fig. 5b, inset). In the low PMS concentration range, the increased reaction rate can be ascribed to the increased concentration of adsorbed PMS 

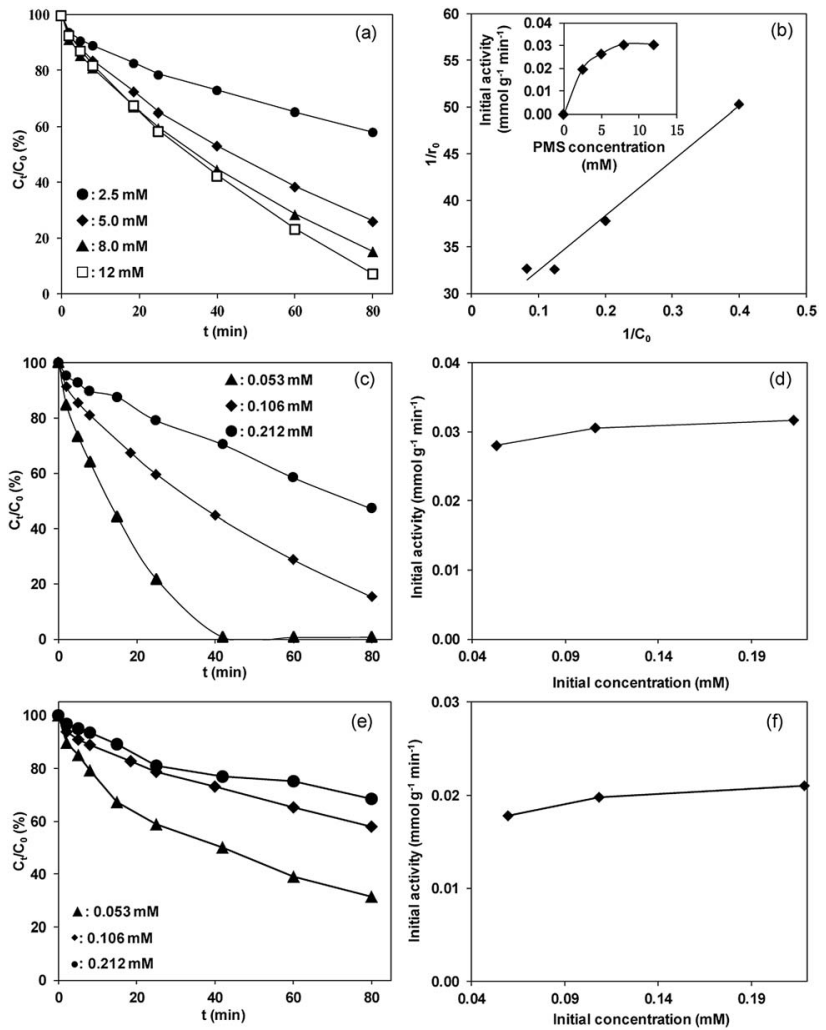

Fig. 5 (a) Effects of initial PMS concentration on phenol degradation, (b) the plot of $1 / r_{0}$ versus $1 / C_{0}$ (inset: effects of initial PMS concentration on the initial activity of CNT); effects of initial phenol concentration on (c) phenol degradation and (d) the initial activity of CNT at a PMS concentration of $8 \mathrm{mM}$; effects of initial phenol concentration on (e) phenol degradation and (f) the initial activity of CNT at a low PMS concentration of $2.5 \mathrm{mM}$. Reaction conditions: catalyst dosage $=0.1 \mathrm{~g}$ $\mathrm{L}^{-1}$.

on catalyst surface with increasing PMS concentration. At PMS concentrations above $8.0 \mathrm{mM}$, the adsorbed PMS concentration remained constant because saturated PMS adsorption was reached, which resulted in nearly identical reaction rates even with further increasing the initial PMS concentration. To confirm the reaction mechanism, the dependency of the initial reaction rate on the initial PMS concentration was further fitted to the Langmuir-Hinshelwood model, ${ }^{45}$

$$
\begin{gathered}
r_{0}=k \theta_{\mathrm{s}}=k \frac{b C_{0}}{1+b C_{0}} \\
\frac{1}{r_{0}}=\frac{1}{k b} \frac{1}{C_{0}}+\frac{1}{k}
\end{gathered}
$$

where $r_{0}$ is the initial phenol degradation rate at the initial PMS concentration $C_{0}, k$ is the reaction rate constant, $\theta_{\mathrm{s}}$ is the surface coverage of PMS adsorption and $b$ is the equilibrium constant for PMS adsorption.

Fig. $5 \mathrm{~b}$ shows the plot of $1 / r_{0}$ versus $1 / C_{0}$. The linear plot of $1 /$ $r_{0}$ versus $1 / C_{0}$ with $R^{2}$ higher than 0.98 showed that phenol degradation on the catalyst obeyed the Langmuir-Hinshelwood model, reflecting that the reaction rate of phenol degradation is controlled by the catalytic activation of pre-adsorbed PMS on the catalyst surface.
To determine the influence of initial phenol concentration, phenol degradation was performed by varying the phenol concentration from 0.053 to $0.212 \mathrm{mM}$ at a fixed PMS concentration of $8.0 \mathrm{mM}$. The results are presented in Fig. 5c. The initial reaction rates of phenol degradation remained nearly constant with increasing phenol concentration from 0.053 to $0.212 \mathrm{mM}$, indicating that the phenol degradation was independent of the initial phenol concentration under our experimental conditions. In parallel, nearly identical reaction rates were observed for phenol degradation with varied initial concentrations at a lower PMS concentration of $2.5 \mathrm{mM}$ (results presented in Fig. 5e and f), confirming the minor impact of the initial phenol concentration on phenol degradation.

In conclusion, the catalytic PMS activation for phenol degradation led to decreased contents of active sites on CNT750 and NCNT-750 due to the increased acidic O-containing groups and decreased basic groups/sites resulting from surface oxidation. Additionally, the interaction between PMS and the catalyst played a crucial role in phenol degradation because reactive species were formed by the catalytic activation of adsorbed PMS. The PZCs of the fresh CNT-750 and NCNT-750 samples were 7.3 and 7.9, respectively. Considering that PMS is present as anions under the experimental conditions, electrostatic attractive interactions between PMS and the fresh catalysts can be expected, leading to strong PMS adsorption and, thus, high catalytic activity. In contrast, the PZCs of U-CNT-1 and U-NCNT-1 were 3.7 and 5.4, respectively. Hence, electrostatic repulsion interactions occurred between PMS and the used catalysts, which suppressed PMS adsorption and the access of PMS to the active sites; this also accounts for the markedly decreased catalytic activities of the used catalysts.

\subsection{Catalyst regeneration}

The catalytic PMS activation led to surface oxidation and formation of O-containing functionalities on both CNT-750 and NCNT-750, as reflected by XPS analysis, PZC determination and Boehm titration. As a result, decreased catalytic activities for phenol degradation were obtained on used CNT-750 and NCNT750 due to their substantially decreased active sites and suppressed PMS adsorption. Accordingly, removal of the Ocontaining functionalities may restore the catalytic activity. Hence, the catalysts were regenerated by thermal treatment and chemical reduction, and the results are presented in Fig. 6a. For CNT, regeneration of U-CNT-1 by $\mathrm{NaBH}_{4}$ reduction led to an increase of the initial activity from 0.01 to $0.018 \mathrm{mmol} \mathrm{g}_{\mathrm{Cat}^{-}}{ }^{-}$ ${ }^{-1} \min ^{-1}$. More effective regeneration was achieved by thermal regeneration, and the catalytic activity of the regenerated CNT was found to be strongly dependent on the thermal regeneration temperature. For example, increasing the thermal treatment temperature from $350{ }^{\circ} \mathrm{C}$ to $750{ }^{\circ} \mathrm{C}$ caused an increase of the reaction rate from 0.021 to $0.059 \mathrm{mmol} \mathrm{g}_{\mathrm{Cat}}{ }^{-1} \mathrm{~min}^{-1}$.

The effective restoration of the catalytic activity can be readily explained in terms of the surface chemistry of the regenerated catalysts. After thermal regeneration, the PZCs were determined to be 5.9, 7.2, and 7.6 for T-CNT-350, T-CNT-550, and T-CNT-750, respectively, which are much higher than that 

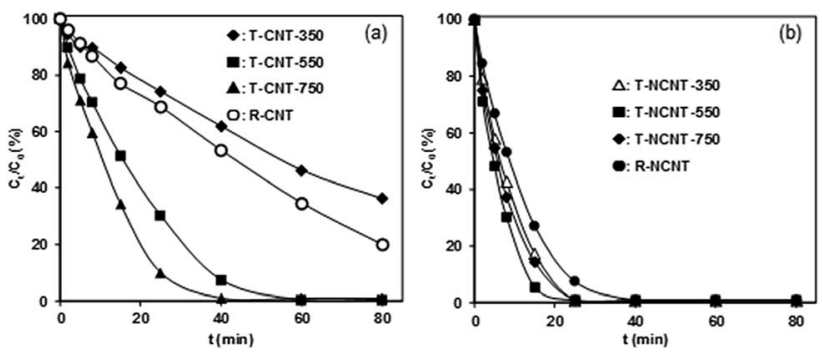

Fig. 6 Phenol degradation on regenerated (a) CNT and (b) NCNT catalysts. Reaction conditions: $[\mathrm{PMS}]_{0}=8 \mathrm{mM}$; [phenol $]_{0}=0.106 \mathrm{mM}$; catalyst dosage $=0.1 \mathrm{~g} \mathrm{~L}^{-1}$.

of the used CNT. The increased PZC of CNT with increasing thermal treatment temperature clearly reveals the effective removal of negatively charged O-containing groups. Accordingly, XPS characterization showed that thermal treatment of used $\mathrm{CNT}$ at $750{ }^{\circ} \mathrm{C}$ led to a decrease of the $\mathrm{O}$ content from 3.50 to 2.39 at\%, mainly contributed by the removal of surface Ocontaining groups. In parallel, Boehm titration results revealed that thermal treatment resulted in increased content of basic groups at the expense of acidic groups. The effective removal of O-containing groups and the formation of Lewis basic sites restored the active sites and improved PMS adsorption on the catalyst surface, giving rise to enhanced catalytic activity.

In comparison with CNT, different regeneration behavior of NCNT was observed (see Fig. 6b). In general, chemical reduction or thermal treatment of used NCNT-750 was capable of restoring its catalytic activity. For example, chemical reduction of used NCNT-750 by $\mathrm{NaBH}_{4}$ led to an increase of the initial activity from 0.051 to $0.078 \mathrm{mmol} \mathrm{g}_{\mathrm{Cat}}{ }^{-1} \mathrm{~min}^{-1}$. In parallel, thermal regeneration of U-NCNT- 1 at $350{ }^{\circ} \mathrm{C}, 550{ }^{\circ} \mathrm{C}$, and $750{ }^{\circ} \mathrm{C}$ resulted in increased catalytic activities of $0.098,0.12$ and $0.11 \mathrm{mmol} \mathrm{g}_{\text {Cat }}{ }^{-1} \mathrm{~min}^{-1}$, respectively. In contrast to CNT, the catalytic activities of regenerated NCNT were much lower than that of the fresh catalyst $\left(0.18 \mathrm{mmol} \mathrm{g}_{\mathrm{Cat}}{ }^{-1} \mathrm{~min}^{-1}\right)$. Characterization results from XPS and Boehm titration showed that both chemical reduction and thermal treatment of U-NCNT-1 substantially eliminated O-containing functionalities, particularly carboxyl groups. Concurrently, markedly increased basic sites were observed. As a result, the catalytic activities of the regenerated NCNT were partially restored. Notably, the $\mathrm{N}$ contents of NCNT-750, T-NCNT-350, and T-NCNT-550 were determined to be 2.56, 2.30 and 1.90 at\%, respectively. Moreover, the $\mathrm{N}$ content in T-NCNT-750 was even below the determination level, reflecting increased $\mathrm{N}$ loss with regeneration temperature. Considering that pyridinic, pyrrolic and graphitic $\mathrm{N}$ species act as highly active sites for PMS activation, the much lower catalytic activities of the regenerated catalysts can thus be attributed to the $\mathrm{N}$ loss during the regeneration process.

\section{Conclusions}

In the present study, the reuse, deactivation and regeneration of CNT and NCNT catalysts in the catalytic activation of PMS were studied, and the variation of the surface functionalities of the catalysts was investigated. The results show that both CNT and NCNT are highly active catalysts in catalytic PMS activation for phenol degradation, while marked catalyst deactivation was observed on the used catalysts. The catalyst deactivation was predominantly due to the formation of O-containing functionalities and decreased PZC, which substantially decreases the active sites of the catalyst and suppresses access of PMS to the active sites due to electrostatic repulsion interactions. Consequently, markedly low catalytic activities are obtained on used CNT and NCNT. Additionally, phenol degradation is highly dependent on PMS concentration, following the LangmuirHinshelwood model; this indicates that the catalytic activation of adsorbed PMS is the rate-controlling step. Chemical reduction and thermal treatment of used CNT and NCNT can remove surface O-containing groups, restoring their catalytic activities. More effective regeneration can be achieved using thermal treatment compared to chemical reduction, and the regeneration effect of thermal treatment is strongly dependent on the treatment temperature. For CNT, the regenerated catalyst exhibits even higher catalytic activity than the fresh catalyst. For NCNT, however, thermal treatment regeneration leads to a marked loss of $\mathrm{N}$-containing functionalities and even to complete $\mathrm{N}$ loss at $750{ }^{\circ} \mathrm{C}$, giving rise to lower catalytic activity than that of the fresh catalyst. These results show that the surface functionalities of CNT and NCNT vary during catalyst reuse, deactivation and regeneration, which are strongly correlated to the catalytic activities of CNT and NCNT for PMS activation.

\section{Conflicts of interest}

There are no conflicts to declare.

\section{Acknowledgements}

The financial support from the National Natural Science Foundation of China (no. 21507056, 21407074 and 21577056) and the Natural Science Foundation of Jiangsu Province (BK20150568) is gratefully acknowledged.

\section{Notes and references}

1 S. K. Ling, S. B. Wang and Y. L. Peng, J. Hazard. Mater., 2010, 178, 385-389.

2 H. Lee, H. J. Lee, J. Jeong, J. Lee, N.-B. Park and C. Lee, Chem. Eng. J., 2015, 266, 28-33.

3 C. J. Liang and H. W. Su, Ind. Eng. Chem. Res., 2009, 48, 55585562.

4 J. M. Monteagudo, A. Durán, R. González and A. J. Expósito, Appl. Catal., B, 2015, 176-177, 120-129.

5 Z. H. Wang, L. Y. Sun, X. Y. Lou, F. Yang, M. Feng and J. S. Liu, J. Colloid Interface Sci., 2017, 507, 51-58.

6 Y. T. Lin, C. J. Liang and J. H. Chen, Chemosphere, 2011, 82, 1168-1172.

7 S. N. Su, W. L. Guo, C. L. Yi, Y. Q. Leng and Z. M. Ma, Ultrason. Sonochem., 2012, 19, 469-474. 
8 T. Zeng, X. L. Zhang, S. H. Wang, H. Y. Niu and Y. Q. Cai, Environ. Sci. Technol., 2015, 49, 2350-2357.

9 Y. S. Zhao, C. Sun, J. Q. Sun and R. Zhou, Sep. Purif. Technol., 2015, 142, 182-188.

10 D. D. Tang, G. K. Zhang and S. Guo, J. Colloid Interface Sci., 2015, 454, 44-51.

11 Y. J. Yao, H. Chen, C. Lian, F. Y. Wei, D. W. Zhang, G. D. Wu, B. J. Chen and S. B. Wang, J. Hazard. Mater., 2016, 314, 129139.

12 S. Indrawirawan, H. Q. Sun, X. G. Duan and S. B. Wang, Appl. Catal., B, 2015, 179, 352-362.

13 X. G. Duan, C. Su, L. Zhou, H. Q. Sun, A. Suvorova, T. Odedairo, Z. H. Zhu, Z. P. Shao and S. B. Wang, Appl. Catal., B, 2016, 194, 7-15.

14 X. G. Duan, Z. M. Ao, D. G. Li, H. Q. Sun, L. Zhou, A. Suvorova, M. Saunders, G. X. Wang and S. B. Wang, Carbon, 2016, 103, 404-411.

15 X. G. Duan, K. O'Donnell, H. Q. Sun, Y. X. Wang and S. B. Wang, Small, 2015, 11, 3036-3044.

16 X. G. Duan, H. Q. Sun, Y. X. Wang, J. Kang and S. B. Wang, ACS Catal., 2015, 5, 553-559.

17 X. G. Duan, H. Q. Sun, J. Kang, Y. X. Wang, S. Indrawirawan and S. B. Wang, ACS Catal., 2015, 5, 4629-4636.

18 H. Q. Sun, S. Z. Liu, G. L. Zhou, H. M. Ang, M. O. Tadé and S. B. Wang, ACS Appl. Mater. Interfaces, 2012, 4, 5466-5471.

19 Y. X. Wang, Z. M. Ao, H. Q. Sun, X. G. Duan and S. B. Wang, Appl. Catal., B, 2016, 198, 295-302.

20 H. Q. Sun, C. K. Kwan, A. Suvorova, H. M. Ang, M. O. Tadé and S. B. Wang, Appl. Catal., B, 2014, 154-155, 134-141.

21 X. G. Duan, Z. M. Ao, H. Q. Sun, S. Indrawirawan, Y. X. Wang, J. Kang, F. L. Liang, Z. H. Zhu and S. B. Wang, ACS Appl. Mater. Interfaces, 2015, 7, 4169-4178.

22 C. Wang, J. Kang, H. Q. Sun, H. M. Ang, M. O. Tadé and S. B. Wang, Carbon, 2016, 102, 279-287.

23 C. Lu and H. Chiu, Chem. Eng. Sci., 2016, 61, 1138-1145.

24 J. Vakros, C. Kordulis and A. Lycourghiotis, Chem. Commun., 2002, 1980-1981.

25 K. Bourikas, J. Vakros, C. Kordulis and A. Lycourghiotis, J. Phys. Chem. B, 2003, 107, 9441-9451.

26 Y. Otake and R. G. Jenkins, Carbon, 1993, 31, 109-121.
27 J. A. Menéndez, J. Phillips, B. Xia and L. R. Radovic, Langmuir, 1996, 12, 4404-4410.

28 Y. X. Wang, Y. B. Xie, H. Q. Sun, J. D. Xiao, H. B. Cao and S. B. Wang, ACS Appl. Mater. Interfaces, 2016, 8, 9710-9720.

29 U. Zielke, K. J. Huttinger and W. P. Hoffman, Carbon, 1996, 34, 983-998.

30 Q. L. Zhuang, T. Kyotany and A. Tomita, Carbon, 1994, 32, 539-540.

31 J. L. Figueiredo, M. F. R. Pereira, M. M. A. Freitas and J. J. M. Órfão, Carbon, 1999, 37, 1379-1389.

32 R. Arrigo, M. Haevecker, S. Wrabetz, R. Blume, M. Lerch, J. McGregor, E. P. J. Parrott, J. A. Zeitler and L. F. Gladden, J. Am. Chem. Soc., 2010, 132, 9616-9630.

33 J. B. Chen, L. M. Zhang, T. Y. Huang, W. W. Li, Y. Wang and Z. M. Wang, J. Hazard. Mater., 2016, 320, 571-580.

34 H. Q. Sun, Y. X. Wang, S. Z. Liu, L. Ge, L. Wang, Z. H. Zhu and S. B. Wang, Chem. Commun., 2013, 49, 9914-9916.

35 H. Chen and K. C. Carroll, Environ. Pollut., 2016, 215, 96-102. 36 H. Lee, H.-I. Kim, S. Weon, W. Choi, Y. S. Hwang, J. Seo, C. Lee and J.-H. Kim, Environ. Sci. Technol., 2016, 50, 10134-10142.

37 H. Zhang, J. Joseph, M. Gurney, D. Becker and B. Kalyanaraman, J. Biol. Chem., 2002, 277, 1013-1020.

38 M. Y. Wei, L. Gao, J. Li, J. Fang, W. X. Cai, X. X. Li and A. H. Xu, J. Hazard. Mater., 2016, 316, 60-68.

39 R. A. Floyd and L. M. Soong, Biochem. Biophys. Res. Commun., 1977, 74, 79-84.

40 J. Y. Yao, X. L. Zeng and Z. Y. Wang, Chem. Eng. J., 2017, 330, 345-354.

41 X. X. Pan, J. Chen, N. N. Wu, Y. M. Qi, X. X. Xu, J. L. Ge, X. H. Wang, C. G. Li, R. J. Qu, V. K. Sharma and Z. Y. Wang, Water Res., 2018, 143, 176-187.

42 X. Cheng, H. G. Guo, Y. L. Zhang, X. Wu and Y. Liu, Water Res., 2017, 113, 80-88.

43 S. D. Yan, W. H. Xiong, S. Y. Xing, Y. Q. Shao, R. Guo and H. Zhang, Sci. Total Environ., 2017, 599-600, 1181-1190.

44 H. Chen, Z. Y. Xu, H. Q. Wan, J. Z. Zheng, D. Q. Yin and S. R. Zheng, Appl. Catal., B, 2010, 96, 307-313.

45 Z. M. de Pedro, J. A. Casas, L. M. Gomez-Sainero and J. J. Rodriguez, Appl. Catal., B, 2010, 98, 79-85. 